\title{
El papel de la física en la formación profesional del ingeniero
}

\section{The roll of physics in engineering univesity education}

Jaime Arturo Lagos Figueroa

Fundación Universitaria Católica Lumen Gentium, Cali, Colombia. Facultad de Ingeniería.

Correo electrónico: jlagosf@yahoo.com

\section{Resumen}

En el presente artículo se plantean algunas reflexiones sobre la importancia de las ciencias básicas, particularmente de la física en la formación de un ingeniero. Con el propósito de brindar claridad sobre su delimitación. Todo este estudio sirvió para concluir que el problema de la ambigüedad que, a veces se plantea, es más coyuntural que gnoseológico, pues en contextos de subdesarrollo tecnológico los propósitos se enmarcan en la importación y aplicación de tecnología, en lugar de hacerse en las oportunidades para la investigación y el desarrollo. También, fue posible conocer que la presencia de las ciencias básicas - particularmente de la física, dentro de los procesos formativos- no se ha sido tan visible como en otros campos, donde la aplicación del lenguaje y técnicas matemáticas son más determinantes.

Palabras clave: ingeniería, ciencias básicas, física, educación en ingeniería, enseñanza de la física.

\section{Abstract}

In the present article, some reflections on the importance of the basic sciences, particularly of the physics, in the formation of an engineer are presented. In order to provide clarity on its delimitation. All this study served to conclude that the problem of ambiguity, which sometimes arises, is more conjunctural than gnoseological, because in contexts of technological underdevelopment purposes are framed in the import and application of technology, rather than in the opportunities for research and development. Also, it was possible to know that the presence of the basic sciences - particularly of phys- 
ics, within the formative processes- has not been as visible as in other fields, where the application of language and mathematical techniques are more determinant.

Keywords: engineering, basic science, physics, engineering education, teaching physics.

\section{Introducción}

Hablar sobre la formación de ingenieros genera muchas reflexiones, más aun cuando la sociedad requiere de un profesional de alta calidad, especialista e investigador, con capacidad de adaptación a las exigencias del mañana. Dentro de este campo, se estructuran tres grandes franjas, en la formación profesional de la ingeniería: la Fundamentación Científica, la Fundamentación en Procesos de Ingeniería y la Formación Social y Humanística.

Dentro de las comunidades de ingenieros, frecuentemente, se dan muchos debates y reflexiones acerca de los orígenes y la pertinencia de las ciencias básicas, en el ámbito formativo. Las dudas surgen, pues, en el momento de la aplicación y el ejercicio profesional de la ingeniería, las relaciones disciplinares -con áreas como la física o las matemáticas- no se hacen tan evidentes; por lo menos, en la mayoría de las ingenierías particulares. Lo que sí ocurre en casos como la Ingeniería Electrónica, por citar un ejemplo. Esto lleva a discusiones, respecto al hecho de que una ingeniería se considera como tal, si aplica o no directamente la química, la física y las matemáticas (tradicionalmente, la ingeniería se ha relacionado con la aplicación de las ciencias básicas en la solución de problemas).

En este estudio, se hace énfasis en la fundamentación científica del ingeniero. Entiéndase como el estudio de las ciencias básicas -íntimamente relacionadas con las ciencias naturales o fácticas, la matemática y la lógica; disciplinas propias de la ingeniería. En particular, la física, cuyo estudio debe enfocarse en el análisis y conceptualización de los orígenes y naturaleza de sus leyes y principios. Para lo cual, se debe utilizar un proceso pedagógico conducente a la asimilación consciente del conocimiento, y no de una simple transmisión y operación.
La preocupación -por la manera como se han venido desarrollando los cursos de física, en las diferentes facultades de ingeniería- lleva a reflexionar sobre la verdadera importancia que tiene esta asignatura en la formación del futuro ingeniero. En la mayoría de los casos, el estudiante de ingeniería y, lo que es más grave, el profesional en ingeniería, desconocen o no tienen muy clara la necesidad de recibir o haber recibido un curso en esta área.

Considerando que la ingeniería es un puente entre la ciencia y la tecnología, se necesitan aquellas herramientas de la física, para que dicho puente se realice. Por esta razón, para un ingeniero, la física debe ser más real y práctica: enfocada en el modo de producción y de vida de la sociedad. Hablar de formación en física, para los ingenieros, es un propósito tanto formativo como instrumental. Porque su objetivo está direccionado en entender la realidad del mundo físico y los principios científicos sobre los que descansa la actividad profesional de la ingeniería. Simultáneamente, hay que dotar al estudiante de unas herramientas y técnicas de análisis y trabajo que le permitan desempeñarse como ingeniero.

\section{Bases teóricas}

\section{Las ciencias básicas, y particularmente de la física, en la ingeniería}

La ingeniería ha adquirido gran auge durante el presente siglo, y nuestro país no ha sido la excepción. En buena parte, porque la actividad del ingeniero tiene relación directa con su formación predominantemente técnica; por tanto, son obvios sus vínculos con el desarrollo tecnológico y de los procesos industriales. A la luz de esta concepción, resulta enorme la importancia del ingeniero, puesto que una de sus misiones es servir de enlace entre la ciencia y la técnica. Su preparación -en matemáticas y física (en general, en ciencias naturales), acompañada con la práctica tecnológica, el desarrollo de aptitudes y los métodos de trabajo adecuados- lo hacen apto para aplicar el conocimiento científico a la solución de problemas relacionados con la industria, los materiales, el uso de los recursos naturales, etc. 
Es urgente que las instituciones de Educación Superior enfoquen los estudios de ingeniería en una formación básica, orientada hacia la tecnología, pero con preparación científica. El pregrado cubre muchas áreas y asignaturas, por esto, no se profundiza. La disciplina, los métodos de trabajo y los criterios resultan de mayor calidad, cuando la superficialidad y la simple acumulación de conocimientos son sustituidas por la profundización en temas básicos tanto en lo científico como en lo profesional. Esta ingeniería básica debe permitir al futuro profesional adaptarse a demandas profesionales distintas y a situaciones nuevas del cambiante mundo en que le tocará desenvolverse.

Así pues, la preparación científica del ingeniero no debe descuidarse; porque la revolución científica - tecnológica del mundo actual está exigiendo un acelerado desarrollo de nuevos conocimientos, cada vez más refinados y complejos. Dicha preparación científica debe estar a cargo de profesionales en ciencias básicas (matemáticos, físicos, químicos, biólogos). Claro está que estos profesionales deben tener en cuenta que la ciencia básica que imparten está destinada a estudiantes de ingeniería y que, por lo tanto, debe haber una excelente coordinación entre los niveles básico y profesional de la respectiva carrera. Estos profesionales, de alguna manera, deben tener cierto dominio de todas las asignaturas que conforman las ciencias básicas en ingeniería.

La física y la ingeniería son disciplinas que históricamente han estado fuertemente relacionadas entre sí. Una reflexión muy general sobre el estado actual de la física y de la ingeniería en Colombia sugiere que ambas disciplinas se enfrentan a problemas comunes en su desarrollo; problemas relacionados con la docencia, investigación, vinculación con el aparato productivo y con políticas de desarrollo científico y tecnológico. Por cierto, estos puntos estos que han sido tratados en innumerables congresos nacionales e internacionales. Por ejemplo, los que periódicamente realiza la Asociación Colombiana de Facultades de Ingeniería (ACOFI).

Por ende, es posible afirmar que las matemáticas, la química, y, en particular, la física son absolutamente indispensables y esenciales para el ingeniero, ya que lo proveen de una visión del mundo y de unas bases científicas y metodológicas para la comprensión de los fenómenos que encontrará, y de las técnicas que deberá practicar en el desarrollo de su actividad profesional.

El desarrollo curricular de estas disciplinas se apoya en un paradigma compartido por un número considerable de científicos e ingenieros, para quienes ha sido exitoso. Pero, ¿será que esto significa que el modelo del mundo físico, de la ciencia y de la técnica que se viene transmitiendo a los estudiantes deba permanecer inalterado? Sin duda, la enseñanza -de las ciencias básicas, y dentro de ellas la Física, en las facultades de Ingeniería- debe ser dinámica e incorporar los avances e innovaciones que se van dando en el paso del tiempo. Para ello, es necesario que los docentes estén actualizados no solo en los saberes propios de su materia, sino en los conocimientos didácticos y pedagógicos. Más aún, deben incorporar, en sus prácticas docentes, el uso de las TIC. ¿se está haciendo, en este sentido, el esfuerzo suficiente?, ¿se cuenta con las herramientas y nuevas tecnologías de información y comunicación necesarias para estar al día?, ¿el docente dedica parte del tiempo a mejorar y actualizar las clases, o simplemente se mantiene en un estado estático y, por lo tanto, desactualizado?, ¿se genera en los estudiantes la curiosidad y el espíritu investigativo? Estos interrogantes nos llevan a reflexionar sobre los verdaderos objetivos de las asignaturas que conforman las llamadas ciencias básicas.

\section{Finalidad de la física}

Carl Sagan, en la introducción del libro Historia del Tiempo de Stephen W. Hawking, plantea una interesante reflexión acerca de lo que debería ser la finalidad de la física:

Nos movemos en nuestro ambiente diario sin entender casi nada acerca del mundo. Dedicamos poco tiempo a pensar en el mecanismo que genera la luz solar que hace posible la vida, en la gravedad que nos ata a la tierra y que de otra forma nos lanzaría al espacio, o en los átomos de los que estamos constituidos y de cuya estabilidad dependemos de manera fundamental... pocos de nosotros dedicamos tiempo a preguntarnos por qué la naturaleza es de la forma que es, de dónde surgió el cosmos, o si siempre estuvo aquí, si 
el tiempo correrá en sentido contrario algún día y los efectos precederán a las causas, o si existen límites fundamentales acerca de lo que los humanos pueden saber... (Sagan, 1988)

Desde el hombre primitivo -que aprendió a utilizar un tronco como arma defensiva, descubrió y utilizó el fuego, talló la piedra y posteriormente construyó las civilizaciones egipcias, china, azteca, maya; entre otras, hasta el que conquistó el espacio, controla la energía nuclear y tiene alto grado de desarrollo actual- han transcurrido quizás dos o tres millones de años. A lo largo de este período, la interacción del hombre con la naturaleza ha permitido que, poco a poco, la humanidad imponga su dominio, empleando la técnica y la ciencia. La ciencia hace parte del progreso social de la humanidad y su método se emplea en cualquier área de la investigación y del conocimiento. De hecho, sus explicaciones, en los procesos técnicos, hacen posible el mejoramiento de las condiciones de la humanidad.

De acuerdo con el origen griego, Física significa, de manera general, estudio o ciencia de la naturaleza; por esta razón, debe considerarse como la cabeza de lo que denominamos ciencias naturales. Los físicos, en su intento de describir los fenómenos naturales con exactitud y veracidad, han llegado a límites impensables: el conocimiento actual abarca, desde la descripción de partículas fundamentales microscópicas hasta el nacimiento de las estrellas en el universo. Incluso, han explorado, con una gran probabilidad, lo que aconteció en los primeros instantes del nacimiento de nuestro universo; por citar unos pocos. Esta tarea la comenzaron hace más de dos mil años, los filósofos griegos Demócrito, Epicuro, Aristóteles y Arquímedes; fue continuada después, por científicos como Galileo Galilei, Isaac Newton, James Clerk Maxwell, Albert Einstein, Niels Bohr, Werner Heisenberg, Paul Dirac, Richard Feynman; entre muchos e importantes personajes de la ciencia física.

El conocimiento de las leyes y de los fenómenos físicos constituye un complemento indispensable en la formación cultural del ser humano moderno, no solo en virtud del enorme desarrollo científico y tecnológico actual, sino también porque el mundo de la física nos rodea en todo momento. En efecto, la ciencia física está inmersa en nuestra vida diaria.
El universo físico siempre ha sido un misterio. Al revisar los intentos realizados por el hombre para entender y explicar el universo, nos asombra el progreso que hemos logrado. En efecto, el fascinante e importante mundo de la física sigue siendo el mundo de la investigación y del descubrimiento. Somos curiosos por naturaleza. No nos basta ver, sentir, gustar, oír y oler el mundo natural y sus productos. Se nos hace necesario describirlo, medirlo, explicarlo y construirlo. Para nosotros, es imprescindible investigar, experimentar y preguntar. La búsqueda del conocimiento es una jornada continua, es difícil pero con muchas recompensas. Y todo comienza con la física, he ahí su finalidad.

\section{Física para físicos y Física para ingenieros}

Cuando se analiza la finalidad de la física, resulta claro que es y debe ser una sola; la diferencia radica en lo que cada quien quiera tomar de ella para su provecho. En este sentido, no es válido hablar de la Física para los físicos y la Física para los ingenieros. Sin embargo, debe existir una selección de contenidos, que los ingenieros - de acuerdo con sus necesidades- las consideren como fundamentales, y las cuales constituyan en una base científico-tecnológica para su formación.

La ingeniería aplica las leyes de la naturaleza para resolver problemas, pero dichas leyes tienen que estar modeladas con teorías matemáticas y leyes físicas. Por ejemplo, la Teoría Electromagnética ha hecho posible el desarrollo de las telecomunicaciones modernas, aplicando todo el análisis en sus complejos modelos matemáticos y físicos; sin estos sus comportamientos no se habrían podido aplicar a la ingeniería. Aunque, en esta relación de ciencias básicas e ingeniería hay que aclarar que cada rama de la ingeniería hace énfasis, según su interés científico, en ramas específicas, no en todo el conjunto general de las matemáticas y las ciencias, como la física. La física -además de describir los fenómenos naturales, con mucha exactitud y veracidad- ayuda a desarrollar la lógica, el sentido común y técnicas de pensamiento y creatividad, importantísimas para toda ingeniería; lo que, a su vez, servirá para el desarrollo profesional y de la vida cotidiana. 
Todo lo anterior, conduce a definir la ingeniería así: es la profesión que permite aplicar los conocimientos científicos, para lograr tanto el aprovechamiento y la transformación de los recursos naturales como la información para satisfacer las necesidades individuales y colectivas. También, se puede afirmar que el objetivo fundamental de la ingeniería es la las aplicación práctica de todas las leyes físicas, para el mejoramiento de la vida humana.

En particular, la física es esencial para el ingeniero, ya que lo provee de una visión objetiva del mundo y de unas bases científicas y metodológicas para la comprensión de los fenómenos que encontrará, y de las técnicas que deberá practicar en el desarrollo de su actividad. Haciendo énfasis, nuevamente, en la dependencia mutua de estas dos áreas, es posible asegurar que -del correcto equilibrio entre la intensidad y dedicación, que a cada una de estas disciplinas se les dé- se originarán valores tan apreciables como: la madurez técnica, la independencia tecnológica y la estabilidad económica. Las Instituciones Universitarias han procurado balancear los objetivos a corto plazo, dentro de un proyecto a largo plazo; así, han ido intentando estimular -con mucha fuerza y tenacidad- el desarrollo de las ingenierías y de las ciencias fundamentales en que estas se apoyan. Sin embargo, pocas veces, desgraciadamente, ha sido exitosa esta tarea.

Con un enfoque de este tipo, la llamada Física para Ingenieros deberá ser más de aplicación a los fenómenos reales. El trabajo experimental no puede considerarse como algo separado de la parte teórica en esta área; por el contrario, debe ser una parte integral de ella. De esta manera, la combinación permanente entre teoría y práctica contribuirá a la comprensión de los fenómenos, las leyes y los principios que conducen al desarrollo de la capacidad analítica del estudiante; lo cual le permitirá explicarse el mundo que lo rodea.

Otro ingrediente primordial que proporciona la física, como fundamento científico en la ingeniería, es la investigación resultante de conciliar y articular lo teórico y lo operativo, con lo que usualmente utilizan los investigadores en sus prácticas y actividades investigativas. Este hecho, por lo demás, no es casual ni accidental: responde a uno de los problemas más agudos que debe enfrentar el estudiante de ingeniería en el instante de traducir, en términos operativos, lo que conoce y ha memorizado desde el punto de vista teórico.

\section{Conclusiones}

La incorporación sucesiva de nuevas ideas, en el panorama científico del mundo, está relacionada -en última instancia- con la producción. Es precisamente la producción la que crea las condiciones experimentales que hacen posible delimitar determinadas series de fenómenos, encontrar las leyes que los rigen $\mathrm{y}$, al mismo tiempo, concentrar la atención de los investigadores en una esfera determinada de fenómenos físicos. El desarrollo tanto de la producción como de las formas sociales explica por qué las concepciones físicas, reflejo de la realidad objetiva, han aparecido en determinado tiempo, en determinado medio científico y en forma determinada.

Por su naturaleza, la ingeniería juega un papel fundamental en el desarrollo científico - tecnológico del país. Ciencia y tecnología se unen para hacer del estudiante de Ingeniería un sujeto de cambio social y un agente activo del desarrollo nacional. Para ello, la ciencia y, en particular, la física, asociadas con la tecnología, sugieren interrogantes para la formación del futuro ingeniero.

Dentro de este contexto, se propone un cambio de orientación en la visión pedagógica y metodológica del proceso de enseñanza-aprendizaje de la física y demás áreas de las ciencias básicas. Esto trae, como consecuencia, el desarrollo de cursos formativos y no informativos. Eso sí, sin distorsionar la concepción de estas asignaturas ni generar animadversión, por parte de los estudiantes hacia ellas.

Por otro lado, es necesario resaltar que la formación, en el área básica de los programas de ingeniería, ha venido deteriorándose. De hecho, ha puesto en peligro la fundamentación científica, necesaria para las áreas profesionales. Esto se refleja en la ausencia de investigación, lo cual impide que las universidades participen en Investigación Aplicada para la Industria. Todo esto genera serios daños a la región y al país. 
Es perentorio reformular los contenidos y las formas de transmisión de los mismos. Esto debe ser concertado, preferentemente entre los profesionales y egresados. Como resultado de esta actividad, surgirá una reflexión obligada: la posible creación de un posgrado en Ciencias básicas para ingenieros, destinado a todos aquellos que, de alguna manera, pretenden cambiar el enfoque tradicional educativo de la formación de ingenieros operativos o técnicos, en profesionales investigadores de alta calidad y competitividad.

\section{Referencias}

ACOFI-Asociación Colombiana de Facultades de Ingeniería. (1998). XVIII Reunión de Facultades de Ingeniería Posgrados en Ingeniería. Memorias del evento. 35p.
ACOFI-Asociación Colombiana de Facultades de Ingeniería. (2005). Marco de Fundamentación Conceptual. Especificaciones de Prueba ECAES de Ingeniería de Sistemas. Versión 6, Bogotá, Colombia. $67 \mathrm{p}$.

Halbwachs, F. (1985). La física del profesor entre la física del físico y la física del alumno. Revista de Enseñanza de la Física, 1(2), 77-89. https://revistas.unc.edu.ar/index.php/revistaEF/article/download/15978/15805.

Hawking, S. (1989). Historia del tiempo. Del big bang a los agujeros negros. Lerner Ltda (Eds.). Bogotá, Colombia. 248 p.

Tippens, P. E. (1981). Física, conceptos y aplicaciones. Mc Graw Hill (Eds.). México, D.F., México. 603 p.

Zalamea G., E., París E. R. \& Rodríguez, M. J. (1985). Física. Educar Editores Ltda (Eds.). Bogotá, Colombia. 248 p. 